\title{
Educación, poder y mercado: deconstrucción crítica de los efectos disciplinantes de las TIC en la nueva Escuela del Espectáculo*
}

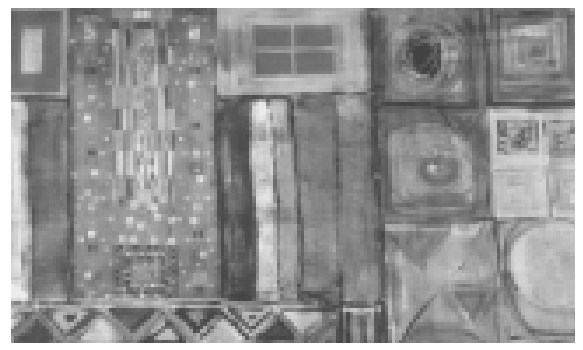

Rafael Vidal Jiménez ${ }^{1}$

JIMÉNEZ, R. V. Education, power and the market: a critical deconstruction of the disciplining effects of Information and Communication Technologies upon the new "Spectacle School". Interface - Comunic., Saúde, Educ., v.9, n.18, p.475-88, set/dez 2005.

Without denying the unquestionable methodological contributions of the new Information and Communication Technologies to the adaptation of teaching methods to the historical changes that are under way, this article encourages self-critical reflection on the possible socio-cognitive consequences of the exaggerated use of these technologies. Basically, it attempts to analyze how this could lead to a triumph of a new aphasic culture: a culture without words, in which the deceptive "hyperrealism" of the ubiquitous Image implies in an iconocratic and iconophagical destruction of verbal language as a mediator, not only of the teaching-learning process, but also of the student-subject relationship with himself and with others. Based on the process of responsibly facing up to post-modern circumstances, what is under discussion here is the rising use of a kind of education spectacle, of education reduced to a mere (consumerist) Imitation of itself, and the loss of words as a vehicle for thought capable of signaling distances with respect to the normalizing diagrams of the new (Disciplinary) Capitalism of Networks and the (Global) Culture of Fear.

KEY WORDS: education. technology. communication.

Sin negar las incuestionables aportaciones metodológicas que pueden representar las TIC - en el contexto de adaptación de la Enseñanza a los cambios históricos que están teniendo lugar-, esta comunicación pretende ser una invitación a la reflexión autocrítica de las posibles consecuencias sociocognitivas de determinado uso "abusivo" de las mismas. Básicamente, se trata de analizar el modo en que ello podría derivar en el triunfo de una nueva cultura afásica: una cultura sin palabras, donde el "hiperrealismo" engañoso de la Imagen omnipresente deviene una destrucción (iconocrática e iconofágica) del lenguaje verbal como mediador no sólo del proceso de enseñanza-aprendizaje, sino de las relaciones del sujeto-alumno consigo mismo y con los demás. Desde el afrontamiento responsable de la situación posmoderna, lo que aquí se cuestiona, en definitiva, es la implantación creciente de una especie de enseñanza-espectáculo, de una enseñanza reducida al mero Simulacro (consumista) de sí, en la pérdida de la Palabra como vehículo de un pensamiento capaz de marcar distancias con respecto a los diagramas normalizadores del nuevo Capitalismo (Disciplinario) de Redes y la Cultura (Global) del Miedo.

PALABRAS CLAVE: educación. tecnología. comunicación.

\footnotetext{
* Ponencia presentada en las II Jornadas Nacionales "TIC y Educación 2005. Tecnologías de la Información y la Comunicación en el ámbito educativo". Lorca, 1-5 de junio de 2005.

${ }^{1}$ Grupo de Investigación en Teoría y Tecnología de la Comunicación, Universidad de Sevilla, Espanha; Facultad de Filosofia y Humanidades, Instituto de Comunicación Social, Universidad Austral de Chile. <1984@terra.es>.

Pagés del Corro, 43, 3ํ B

Sevilla, Espanha

41010
} 


\begin{abstract}
¿Política? ¡Una columna, dos frases, un titular! Luego, en pleno aire, todo desaparece. La mente del hombre gira tan aprisa a impulsos de los editores, explotadores, locutores, que la fuerza centrífuga elimina todo pensamiento innecesario, origen de una pérdida de valioso tiempo.

(Fahrenheit 451, Ray Bradbury)
\end{abstract}

La elocuencia del poder está en el silencio que genera.

(Basil Bernstein)

\title{
Escuela del Espectáculo y hegemonía tecnocapitalista
}

Desde el talante crítico y problematizador que anima este escrito, estimo que cualquier consideración de las relaciones entre las nuevas Tecnologías de la Información y Comunicación (TIC), de un lado, y la Educación, de otro, no debe consistir, en mi opinión, en una mera cuestión metodológica presuntamente neutral, concerniente al perfeccionamiento de los procedimientos destinados a la consecución de los objetivos pedagógicos contemplados en el currículum. Es más, como se deducirá de lo expuesto más abajo, creo que ello constituye un auténtico peligro, puesto que evade la cuestión central que, a mi entender, debe estar en la base de toda discusión sobre la educación: el papel primordial que -como agente socializador de primer orden- juegan los dispositivos pedagógicos en los procesos de producción, reproducción y transformación de la cultura dominante y de las relaciones de poder vinculadas a ella. En la práctica, el debate sobre la educación está íntimamente ligado hoy al modo en que unas "relaciones (categorizadoras, clasificadoras y segmentadoras) de poder" $y$ unas "estrategias de control" - reproductoras de esas relaciones (diferenciales y disimétricas) de poder-se traducen en unos "principios de comunicación" desde los que se dinamizan las posiciones relativas de los individuos y de los colectivos en el conjunto social (conflictivo e inestable) permanentemente configurado.

Convertidas las relaciones sociales en una actividad codificada que selecciona, combina y separa, una de las funciones esenciales de nuestro sistema educativo actual -como el resto de los que imperan en el mundo occidental- es la producción masiva de conocimientos técnico-

administrativos que están acumulando y utilizando últimamente los grupos dominantes del nuevo Capitalismo de cara al afianzamiento de su hegemonía (económico-político-cultural), en un sentido gramsciano. Así, habremos de contemplar la cuestión educativa desde los "enmarcamientos" que hace prevalecer, regulando esos principios comunicativos que gobiernan «la «selección (lo que puede decirse), su progresión, su secuencia, su ritmo, y, crucialmente, los criterioss (Bernstein, 1990, p.41).

El comando pedagógico -propio de lo que denomino Capitalismo (Disciplinario) de Redes- responde, en síntesis, a la manera en que, desde el desarrollo de lógicas de dominación cada vez más complejas e intrincadas, determinadas estrategias generales de poder cristalizan, se concretan $e$ instalan en una nueva Escuela cada vez más permeable al proceso de "desincrustación" y unificación sin fronteras del pragmatismo (egoísta) capitalista [Michéa, 2002]. Ello, en el plano del (atemporal y mítico) Eterno 
${ }^{2}$ Como ya he hecho en otros estudios de base, considero que esta nueva forma de capitalismo global responde a una nueva lógica temporal reactivadora de la premoderna repetición de lo idéntico (Vidal, 2003). Y es que el nuevo Mito Capitalista se ha convertido en una auténtica

«construcción unitaria del pensamiento que garantiza la

permanencia de la totalidad del orden cósmico en torno al orden que la sociedad ya se ha realizado de hecho en el interior de sus fronteras» (Debord, 2002, p.119).
Retorno del Mercado ${ }^{2}$ y de ese consecuente pathos tecnológico que hoy empapa hasta el último rincón de una experiencia humana, así imposible, si concebimos ésta como un auténtico saber a posteriori, ajeno a la tiránica predicción tecnocientífica.

Sin embargo, no podemos caer en el error de una despolitización que nos oculte el verdadero contexto socio-cultural del quehacer docente, ni en la trampa de una desresponsabilización ética que nos impida apreciar las estrategias práctico-discursivas que subyacen en la fijación, organización y puesta en marcha de las nuevas políticas educativas, estimo que hablar de TIC y Enseñanza supone, ante todo, preguntarnos correlativamente por la Escuela que tenemos, por la Escuela que queremos, y, una vez precisadas las distancias existentes entre una y otra, por la(s) Escuela(s) que es(son) posible(s), y bajo qué condiciones. Aprovechemos, así, la ocasión para interrogarnos

$$
\begin{aligned}
& \text { ¿Por qué determinados aspectos de una cultura colectiva aparecen } \\
& \text { en la escuela como conocimiento objetivo? ¿Cómo, en concreto, } \\
& \text { puede el conocimiento oficial representar los fundamentos } \\
& \text { ideológicos de los intereses dominantes de una sociedad? ¿Cómo } \\
& \text { legitima la escuela estos niveles de conocimiento parciales y } \\
& \text { limitados como verdades incuestionables? (Apple, 1997, p.35) }
\end{aligned}
$$

Se trata, en síntesis, de unas cuestiones que nos obligan a pensar el problema educativo desde la óptica de ese fenómeno de objetivación de la subjetividad que, en la filosofía relacional de Michel Foucault, responde a la articulación (dialógica, retroactiva y holográfica) de esos tres aspectos esenciales en torno a los cuales viene girando también mi modesta labor investigadora:

1. la hermenéutica del sí mismo en referencia a las formas de autorreconocimiento de un ser pléctico-emergente, realizado (diferencial, selectiva e intersubjetivamente) como lugar dinámico de cruzamiento/ entrecruzamiento variable de flujos de acción comunicacional múltiples y heterogéneos;

2. el poder como dimensión relacional-inmanente a partir de la cual los sujetos adquieren la capacidad de condicionar y orientar - de manera recíproca, pero desigual - el marco de acciones selectivas que los define, dentro de la esfera social inscrita en su mismo dominio organizador; 3. el saber como relación específica con la "verdad", regulada de acuerdo con las prácticas-discursos desde las que se habilitan en un aquí y ahora (contingentes y no-predeterminados) unos objetos que ni preexisten a ese acto relacional ni son fruto de una creación ex nihilo por parte del lenguaje (Foucault, 1991, 1992).

Los problemas de la identidad - como Paul Ricoeur, prefiero hablar del más complejo concepto de ipseidad (Vidal, 2005), del poder y del saber se conectan aquí, por tanto, con el tema (sistémico-relacional-comunicacional) de los niveles de encaje estructural, o sea, de los parámetros de continuidad-discontinuidad entre esa Escuela que tildo de espectacular, y el desarrollo de lo que se ha venido en Ilamar "Sociedad (Global) de la 
Información". Mi cuestionamiento de los posibles efectos sociocognitivos (disciplinarios, paralizadores y anuladores) sobre la construcción social de un nuevo modelo de sujeto-profesor y sujeto-alumno me lleva a dirigir mis indagaciones hacia la manera en que esa Escuela-Espectáculo - aquélla en la que todas las relaciones afectivas-cognitivas-éticas entre sus integrantes están mediadas, o, mejor, mediatizadas, por la Imagen, en detrimento de la Palabra- es el correlato iconocrático de una Escuela-Mercado, puesta al servicio de la generación de activo potencial consumista, allí donde la consunción obsesivo-compulsiva de bienes superfluos transforman (psicosocialmente) las necesidades secundarias en primarias (o vitales). Defiendo, pues, que las relaciones entre TIC y Educación hay que plantearlas en nuestro presente singular desde la problematización de un nuevo modelo autoorganizado y autosostenido, basado en unas relaciones de poder hegemónicas, que, identificando la nueva verdad tecnocrática con el único saber posible, deriva hacia la reproducción de un tipo de subjetividad asentada en el consumismo como factor de identificación ( $y$ autorreconocimiento) cuasi-exclusivo.

Como señala Ángel San Martín, apoyándose en la obra de Armand Mattelart,

... con independencia de las valoraciones que puedan hacerse del fenómeno de las TI, lo bien cierto es que actúan como hegemónicas en casi todos los órdenes de la vida en las sociedades capitalistas avanzadas. La escuela, en consecuencia, tampoco permanece ajena a esta tendencia, pues constata, no sin cierta perplejidad, cómo las tecnologías van tomando posición en sus espacios. Hecho que, al concebirse como el signo de los tiempos, parece inevitable $y$ hasta molesta su cuestionamiento, aunque desde las TI sí se objeta la pertinencia de mantener un sistema tan caro e ineficaz como el escolar. Un modo de reforzar esta tendencia desregulada se concreta en propugnar una educación por y para los productos culturales de las tecnologías, bajo la impronta publicitaria que identifica el aprendizaje con el entretenimiento, contribuyendo así a la despolitización de aquél o, si se prefiere, a vincularlo a valores ${ }^{3}$ El subrayado es mío. morales globales. (San Martín, 1998, p.109]³

Así es. Estamos ante un preocupante y confuso proceso que nos debe impulsar a tomar conciencia de la creciente interpenetración holográmática que hoy se está produciendo entre las distintas esferas de la vida social; esferas que antes gozaban de cierto grado de autonomía lógica, simbólica y funcional. Como ya he sugerido, el Mercado también está penetrando, invadiendo capilarmente la Escuela, sometiéndola en la generalización planetaria de la Lógica Inmanente del Capital, de ese nuevo modo de Soberanía Global que actúa, más allá de la capacidad de control de los sujetos implicados, en -y no frente a- el mismo plano de las relaciones de dominación-subordinación que ordena (Hardt y Negri, 2000). Lo cual supone la supresión de fronteras fijas entre unos afueras y adentros en 
constante desplazamiento y reemplazamiento estratégico, es decir, afines a los intereses específicos que en cada momento actúan con arreglo a dos pautas de interacción complementarias, intensamente individualistas $e$ insolidarias: el consumismo - como «una forma de vida completa, la obsesiva y patética búsqueda del disfrute siempre diferido del Objeto que falta, reivindicado como tal en la práctica y celebrado en la fantasía como una contracultura emancipadoras (Michéa, 2002, p.35), y el miedo al "otro" como amenaza contra-consumista".

En ello, naturalmente, ejercen una labor canalizadora fundamental las TIC, los Medios Globales, en la medida en que producen y gestionan relaciones irrecíprocas, intransitivas y antimediadoras, sujetas a los imperativos - "incontestables", y de ahí su tremenda violencia simbólica- del CÓDIGO, y de los efectos de limitación de los intercambios comunicativos que éste rige. Lo determinante en los Medios no es su contenido en virtud de su supuesta neutralidad funcional, sino, precisamente, su misma forma de operar, abstrayendo, separando, anticipando, imposibilitando los intercambios. De ahí que los considerase no como meros vehículos portadores de una ideología concreta, no como "coeficientes", sino como "efectuadores" de ideologías, sean las que sean [Baudrillard, 1996]. A mi entender, cada vez parece más plausible la intuición mcluhaniana acerca de la correspondencia entre medios y mensaje, pero en un sentido utópico negativo, opuesto al optimismo determinista tecnológico del propio McLuhan. «El carácter fundamentalmente tautológico del espectáculo asevera Guy Debord-se deriva del hecho simple de que sus medios son, al mismo tiempo, su fins (Debord, 2002, p.41). Y esto cobra una gran importancia en el tipo de Disciplina cognitiva, afectiva y ética que las TIC están empezando a instituir -desde su uso abusivo, masivo y central- en esa nueva Escuela (iconofágica) del Espectáculo, basada en un conjunto de comportamientos interiorizados, ajenos - en la ilusión del yo- a su genealogía dialógica-relacional de cuño infocapitalista, y cuyos rasgos más notables pasaré a describir.

Pero antes de ahondar en ello, haré unas rápidas aclaraciones que me parecen pertinentes en este momento. En primer lugar, no me opongo en ningún caso a la utilización de las TIC como interesantes herramientas de trabajo, siempre que éstas estén subordinadas a lógicas de trabajo sostenidas por la fuerza de la palabra, la gestualidad y la presencialidad como camino hacia un saber autocomprensivo y héterocomprensivo. Yo soy el primero que participo de y aconsejo el uso de una diversidad significativa de medios audiovisuales, de sistemas multimedia e hipermedia, de internet etc., para la consecución de objetivos que van más allá de su propia lógica estetizadora de lo real, hecho que - y quiero resaltarlo - no siempre resulta fácil. Quien interprete mi exposición de acuerdo con el "viejo" debate entre "apocalípticos" e "integrados" no habrá comprendido mis verdaderas intenciones. Lo que me preocupa es que las TIC estén en la base de todo un nuevo "paradigma" ( $y$ "ritual") educativo que haga de la mera contemplación voyerista y del entretenimiento alienador su medio y fin último. El resultado, la invocación de un determinismo tecnológico recuérdese mi subrayado de la cita anterior de Ángel San Martín - que, 
primero, deviene en la desmovilización y la pasividad social resultante de la proclamación de la Técnica como destino inexorable de la Humanidad; y que, segundo, hace del "aula virtual" (y los aparatos técnicos que la constituyen) no ya una prolongación, sino una auténtica sustitución de las facultades sensoriales y cognitivas humanas en tanto, según los delirios utopistas de Mcluhan, «los nuevos medios son ampliaciones macroscópicas de nuestras propias autoamputaciones» (Colina, 2005, p.4) ${ }^{5}$.

En segundo lugar, parece existir una enorme paradoja en la ineludible pregnancia de la Disciplina Global en todo lo tocante a las relaciones (económico-sociales, de género y étnico-culturales) de poder y dominación que organiza. El alcance sojuzgador de las reglas constitutivas del "dispositivo pedagógico" - éste, «en su creación selectiva, posicionamiento y oposición de sujetos pedagógicos, es una regla simbólica de la conciencias (Bernstein, 1990, p.111) ${ }^{6}$ es "independiente" de la intencionalidad transformadora de algunos sujetos implicados. Es más, siempre que no se encuentre una efectiva amplificación de esas líneas de fuga y resistencia con respecto a los "diagramas normalizadores" que dan vida al MetaDispositivo - participado por el propiamente pedagógico-, cualquier gesto individual que represente un acto de desviación frente a sus fuertes "imprintings culturales" operará como fuente de alimentación de la lógica autocorrectora del Sistema. Esta permanente construcción del Orden a partir de esos desórdenes - que dan cuenta de la naturaleza compleja y conflictiva de aquél- coincide, por consiguiente, con una importante discordancia entre las consecuencias de las intervenciones a un nivel microsocial y la reproducción de lo existente a escala macrosocial?.

En tercer y último lugar, a la luz de los nuevos Estudios Culturales, que están en los cimientos de mis motivaciones deconstructivas, los procesos de producción, transmisión y adquisición del discurso-práctica pedagógico no se resuelve, en la práctica, en la simple recepción y adecuación pasiva de los sujetos a los mandatos del Modelo. Más bien, estamos ante una tensión negociadora constante que define la transacción cultural como un complejo juego de adaptaciones y oposiciones diferenciales, desde el que surgen determinadas soluciones hegemónicas. Lo importante es que, como ya lo he hecho entrever, esas fisuras, esas contradicciones constitutivas del Sistema, pueden estar en la base de su posible trasgresión modificadora, en un sentido cualitativo. Sea como fuere, todo análisis que pretendamos hacer de los trasvases disciplinarios que tienen lugar entre la estructura relacional de poder del "dispositivo pedagógico" y el Meta-Orden Global al que pertenece debe tener en cuenta el papel activo que profesores $y$ alumnos desempeñan en la reinterpretación co-creadora de los patrones de interacción dominantes en todos los terrenos afectivos, cognitivos y éticos. Esto, por supuesto, obliga a considerar siempre los mismos planos psicosocio-cognitivos-culturales sobre los que incidirán de manera disimétrica, pero, en general, efectiva, los patrones conductuales hegemónicos.

Velocidad, imagen y simulación

Como ya he adelantado, las exigencias fundamentales que caracterizan lo que propongo llamar Escuela (embrutecedora) del Espectáculo están
${ }^{5}$ En ese sentido, me parece necesario revisar afirmaciones como la que apoya la institucionalización de esa "aula virtual" en la creencia de que «al menos de un modo genérico, los alumnos comprenden mejor algunos conceptos contenidosy

procedimientos ya que los medios audiovisuales les son más familiares a alumnos que acostumbran a recibir más información por estos medios que por cualquier medio escritos (Osset, 2004, p. 60).

${ }^{6}$ Más en concreto, hay que aludir a: 1. Las "reglas (productoras) distributivas", que establecen el control sobre las posibilidades de lo impensable y quién puede pensarlo. 2. Las "reglas (transmisoras) de recontextualización", que construyen el discurso pedagógico como medio de apropiación selectiva y reubicación de otros

discursos, insertando de algún modo el

"discurso de

instrucción”, es decir, el que crea habilidades y competencias

especializadas, en el

"discurso regulativo" referido al mismo

orden social. 3. Las

"reglas de evaluación" de la práctica pedagógica, ligadas a la adquisición del mismo

"texto pedagógico"

[Bernstein, 1990]

${ }^{7}$ Como constata Apple (1997, p.29) citando a

DiMaggio, " "actores

resueltos, razonablesy

bienintencionados" a

menudo contribuyen -

simplemente por

perseguir sus fines

subjetivos - a

mantener estas

relaciones

estructuraless. 
referidas a la negatividad (intrínseca) de ese "accidente" tecnológicoinformacional que autores como Paul Virilio sitúan en las presumibles consecuencias que las Nuevas Tecnologías pueden comportar sobre nuestras relaciones consigo mismo - la ipseidad -, con los demás - el poder, y el mundo - el saber, siempre, por supuesto, que adquieran el grado de centralidad autoreguladora que comienzan hoy a tener. El citado filósofo y urbanista francés nos emplaza, pues, "frente" al inquietante "poder dromocrático" - al poder de la velocidad no sólo vinculado al control sobre el "otro", sino a la acumulación de riqueza derivado de ese control - de un (ciber)-mundo realizado en el tiempo real de las conexiones electromagnéticas a la velocidad de la luz: el poder de los flujos desterritorializadores, que, entrañando la total abolición poshistórica y posgeográfica de los trayectos entre las entradas y las salidas, desemboca en la ubicuidad, la hiperpercepción, la inmediatez y la descorporeización telepresencial de unas relaciones con uno mismo, con el "otro" y con el mundo ahogadas en su propia fugacidad inmaterial (Virilio, 1997). En este nuevo universo cibernético, donde la deslocalización de la comunidad de los presentes corre paralela a la vacía coexistencia virtual de los ausentes, no importa tanto lo transmitido como el simple frenesí (trivializador) de la transmisión; lo que conlleva una enorme dificultad en la construcción transubjetiva de sentido contingente. De este modo, lo virtual anida en ese Gobierno Hiperreal de la Gran Simulación en el que se ve y se mira, pero no se observa, en el que se toca, pero no se siente, en el que se oye, pero nunca se escucha: la absoluta espectacularización, especularización, estetización y banalización de una "realidad" reducida a mercancía lúdica, a objeto de deseo nunca satisfecho en su infinita y falaz repetición.

Las TIC, no como modestos instrumentos subsidiarios, sino como una nueva forma de "(no) estar en el mundo", suponen la inmersión en una nueva cultura que sólo remite a sí misma en la medida en que el signo sólo remite al signo, $y$ la representación es el concepto mismo de lo representado ausente. Identifico una dimensión liberadora en todo esto, en cuanto esta ruptura de las cadenas significantes - en el marco de una desestabilización (posmetafísica) de los conceptos-contenidos - implica la apertura a la posibilidad que siempre es otra, a la autodefinición de nuestra existencia como el genuino laboratorio de una experiencia que se excede a sí misma, más allá de la sujeción a esa Verdad (predictiva) que hoy podemos desmontar en un sentido extramoral, puesto que «las verdades son ilusiones de las que se ha olvidado que lo son; metáforas que se han vuelto gastadas y sin fuerza sensible, monedas que han perdido su troquelado y no son ahora ya considerada como monedas, sino como metal» [Nietzsche, 1994, p.25]. Sin embargo, esas sagradas e intocables tecnologías poseen otra dimensión normalizadora que amenaza con imponer su fortaleza disciplinaria al suplantar la comunicación dialógica por el intercambio (monológico) de informaciones consigo mismo, dentro de una nueva soledad postsocial donde florece la nueva figura épica del "ciberhéroe": el nuevo arquetipo humano que «termina por identificar el referente con su propia práctica, pues la información es indiferente al sentidos (López García, 1998, p.89), que sólo maneja formas que llevan a otras formas de 
manera indefinida, puesto que ese "referente en pantalla" ya no es una realidad cognitiva que, compartida por emisor y receptor, pueda estar abierta a la productividad interpretativo-comprensiva transubjetiva.

En el sentido así expuesto, las TIC nos conducen a nuestra autodesaparición en el desierto intangible de una ausencia absoluta, o, si se quiere, de una presencia fingida. Semejante trastocamiento del "principio de realidad" nos introduce en el laberinto "invisible" de esa "máquina de visión" productora de una "visión sin mirada" (Virilio, 1989); nos abandona al dominio de esos ojos electrónicos que vigilan la adaptación de nuestras actitudes y aptitudes a esa Hiperrepresentación de la que ya no somos dueños. Y es que, como recuerdan Silva y Browne, citando las palabras del pintor Paul Klee, «ahora los objetos nos perciben» (Silva y Browne, 2004, p.156). Son la televisión y el ordenador los que, en la práctica, nos miran a nosotros, y no al contrario, cuando el verdadero acontecimiento es la técnica misma:

... el objeto es ahora el que refracta al sujeto y le impone su presencia y su forma aleatoria, su discontinuidad, su fragmentación, su estereofonía y su instantaneidad artificial. Es la potencia del objeto la que se abre paso a través del propio artificio que le hemos impuesto. (Baudrillard, 1996, p.104)

Semejante inversión de los roles de sujeto-objeto entre la máquina y el hombre sólo "acontece" (performativamente) en el espectáculo, ya que, siendo éste

... el desvanecimiento de la distinción entre el yo y el mundo por destrucción del yo asediado por la presencia-ausencia del mundo, es también el desvanecimiento de la distinción entre lo verdadero $y$ falso por represión de toda verdad vivida en beneficio de la presencia real de la falsedad que garantiza la organización de las apariencias. (Debord, 2002, p.174-5)

Es esa instancia auto-simuladora la que ha terminado por convertir el mundo en esa fábula anunciada por Nietzsche; en ese Show (Global) de Truman en el que todos somos espectáculo programado en directo las veinticuatro horas del día.

La gestión empresarial de la Escuela del Espectáculo En la novela de anticipación "Fahrenheit 451", Ray Bradbury nos introduce en la pesadilla de un mundo imaginario -inundado de antenas y pantallas de televisión- donde, en consecuencia, el autismo sobreperceptivo de sus personajes es el correlato de la condena de la lectura como delito, como la más grave amenaza contra una "armonía" social tan sólo alterada por los sonidos de sirena que alertan al cuerpo de policías-bomberos sobre la peligrosa localización de algún arsenal residual de libros, dispuestos a ser consumidos en las llamas purificadoras del fuego salvador y pacificador. En realidad, ese mundo está entre nosotros, está en el espectáculo como 
prohibición tácita del libre pensamiento, del lenguaje verbal, del diálogo, del intercambio cuerpo a cuerpo, piel a piel, justamente porque todo ello sólo es un objeto "inexperimentable". La nueva Escuela ya se encarga de fabricar ese último "hombre cibernético", al que - tras las promesas del falso progresismo imperante- le espera un no-futuro plenamente planificado. Cuando el nuevo modo de gestión de los centros docentes va adquiriendo un sentido cada vez más empresarial, la enseñanza-espectáculo atiende a unos objetivos bien ajustados a las necesidades autorrealizadoras del Mercado. Para empezar - eliminando las últimas posibilidades de un aprendizaje crítico y responsable, capaz de apreciar los intereses encubiertos por la cegadora luminosidad electrónica-, primero, el entretenimiento embrutecedor, segundo, la formación en los valores agresivos del egoísmo insolidario (y posesivo) y la desconfianza en el "otro"- $y$, tercero, la universalización de una ética consumista -tan sólo concretable en el señuelo de un deseo deseante tan sometedor, como irrealizable-, son los nuevos objetivos de una Escuela pública que se está preparando para la contención estrategia de una mayoría excedentaria, cuya inutilidad socio-laboral está bien prevista por la Lógica del Capitalismo (Disciplinario) de Redes.

Pero vayamos por partes. En los escalones intermedios de la formación técnico-profesional, los procesos de enseñanza-aprendizaje de la ignorancia se basan en saberes, en principio, desechables y rutinarios, tan sólo adaptados a contextos tecnológicos muy concretos, que, no obstante, son reaprovechados desde una perspectiva capitalista, como conocimientos utilitarios, de naturaleza algorítmica, que ni siquiera exigen ningún grado de autonomía y creatividad por parte del cibereducando. En cualquier caso, la banal dotación tecnológica de nuestras espectaculares escuelas representa infinitas posibilidades para la colonización mercantil de los grandes corporaciones transnacionales, de las grandes marcas, dispuestas a vender a todos sus participantes, una vez convertidas «en colaboradoras explícitas del "acto educativo"» (Michéa, 2002, p.45). Por consiguiente, si nos situamos en el ángulo de la empresa soberana, esa banalidad (camuflada curricularmente) no lo es tanto, más todavía cuando el aprendizaje de ese número limitado de habilidades responde a intereses técnicos directamente relacionados con el proceso así descrito por Michael Apple (1997, p.165-6):

$$
\begin{aligned}
& \text {... en la sociedad en general, las personas consumen como } \\
& \text { individuos aislados. Su valor está determinado por la posesión de } \\
& \text { bienes materiales o, como decía Will Wright, de destrezas técnicas. } \\
& \text { La acumulación de tales bienes o del "capital cultural" de } \\
& \text { competencia técnica -porciones atomizadas de conocimiento y } \\
& \text { habilidades medidas a través de exámenes y evaluaciones- es un } \\
& \text { procedimiento técnico que precisa únicamente del predominio de } \\
& \text { las destrezas técnicas prioritarias y suficiente tiempo para seguir } \\
& \text { las reglas, al ritmo personal, hasta su conclusión. }
\end{aligned}
$$

En el escalón inferior, el de la inmensa masa de "superfluos", para los que el Sistema les reserva el destino del desempleo - o del empleo precario temporal-, las Nuevas tecnologías alcanzan su cenit espectacularizador, 
toda vez que tienen como única función la disolución de cualquier rastro de ese conocimiento lógico que permitiera la adquisición de la facultad de separar el grano de la paja, y, de paso, facilitase la oposición (transgresora) de la inteligencia a las patrañas hegemónicas. Como punto de inserción entre el Mercado y la Escuela, la Tecnología legitima el Sistema en tanto actúa como nueva imagen de marca de la supuesta modernización y universalización de una "enseñanza de calidad" para todos. Pero, en realidad, la cuestión no pasa -sin olvidar la brecha tecnológica que separa a una institución escolar muy jerarquizada en cuanto al acceso desigual a las dotaciones técnicas- de la puesta a punto de un mecanismo de control que compagina el vulgar entertainment con la instalación de esa nueva "jaula de hierro" weberiana relacionada con la colocación "en la entrada de ese gran parque de atracciones escolares algunos dispositivos electrónicos muy sencillos para detectar la presencia eventual de objetos metálicos» (Michéa, 2002, p.45).

En el otro extremo, la atención docente a las futuras elites camaleónicas encargadas de tomar el testigo en la dirección tecno-gestora-financiera del Capitalismo de Redes, requiere los mayores esfuerzos en la inversión en un capital humano formado según las condiciones más exigentes y tradicionales de un currículum que, en todo caso, se cuida mucho de fomentar en exceso a favor de un saber "útil" y "simplificado" en cuanto especializado, fragmentado, $y$ ajeno a cualquier tipo de enlace contextual en series de pensamiento complejo- esos conocimientos amplios, reflexivos y críticos que sólo pueden ser la fuente de la disidencia. En 1980, el historiador francés Alain Guerreau hacía una inteligente denuncia de las graves deficiencias detectadas en la docencia de la historia, dentro de la enseñanza secundaria de su país, en la siguiente dirección:

$$
\begin{aligned}
& \text {... puede parecer inevitable que para “explicar” las cruzadas a los } \\
& \text { chicos se esté obligando a utilizar la lógica del western, pero que } \\
& \text { la segunda guerra mundial sea concebida en términos tanto } \\
& \text { psicológicos como atomistas de un western a escala mundial para } \\
& \text { alumnos de diecisiete y dieciochos años, los cuales no volverán a } \\
& \text { seguir ningún curso de historia en el futuro, es algo que debería } \\
& \text { suscitar serios problemas y, sin embargo, nunca he oído el más } \\
& \text { mínimo comentario sobre la cuestión a mis colegas. En esas } \\
& \text { condiciones, ¿por qué extrañarse de que la investigación histórica } \\
& \text { sea concebida únicamente como una novela policíaca y de que los } \\
& \text { archivos estén plagados de genealogistas? (Guerreau, 1984, p.19) }
\end{aligned}
$$

Mucho me temo que esta es la meta final de la enseñanza, en general, dentro de esa nueva Disneylandia escolar, lista para rentabilizar económica $e$ ideológicamente las andanadas sucesivas de "webquests", "juegos on-line" y demás curiosidades técnicas de última generación.

Así las cosas, este fetichismo tecnológico no tendrá otro resultado que la constitución de una sociedad amnésica, sin referencias de pasado y futuro, inmovilizada, incapaz de habilitar esos horizontes (móviles) de la experiencia y de las expectativas como principios de acción de un presente 
comprometido con la superación de sus propias condiciones de posibilidad. El problema principal que presenta la perspectiva de la incipiente Escuela del Espectáculo está, pues, en el modelo cognitivo-operacional que instaura; un modelo que se basa en la ilusión de una respuesta, en realidad, contenida en las propias condiciones de los interrogantes planteados. Lo decisivo es la producción en serie de sujetos flexibles, acomodaticios y previsibles en sus comportamientos triviales. En nuestros sistemas educativos actuales,

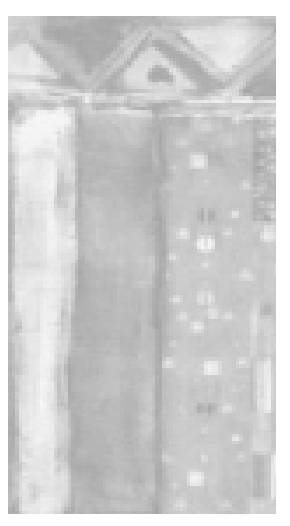

Pero las consecuencias de la nueva alianza (disciplinadora) entre TIC y Enseñanza que aquí estoy cuestionando no se reducen al ámbito del nuevo ciberalumno. Las reformas educativas que se están llevando a cabo en el mundo occidental - enarbolando los principios empresariales (legitimadores) de la eficacia, la racionalización y la rentabilidad como criterios reguladores primordiales- prestan una especial atención al establecimiento de un nuevo modelo de gestión laboral, que está desembocando en un incremento paulatino de la vigilancia y centralización del trabajo del profesorado. A la vez que, en muchos casos, se está viendo obligado a abandonar su papel tradicional de transmisor de conocimientos - a cambio de su reciclaje como animador sociocultural y administrador del espectáculo, cuando no de puro controlador, distribuidor y clasificador de cuerpos-, parece constatable la progresiva pérdida de la competencia selectiva del docente en la elaboración tanto de los programas como de los planteamientos pedagógicos, redirigidos tecnológicamente. Este doble proceso de descalificación y recalificación que, a mi entender, está en la base del enorme malestar que afecta hoy a la profesión, va acompañado, pues, de una cada vez más apreciable separación (pseudo-fabril) entre concepción y ejecución del trabajo (Apple, 1997). El traslado paulatino de la lógica gestora empresarial a la Escuela termina por concretarse en el afianzamiento de la iniciativa privada en los centros de enseñanza mediante la imposición por parte de la administración pública mediadora de programas de gestión informática -personalmente, puedo dar cuenta del que actualmente está operando en el ámbito de competencias de la Consejería de Educación y Ciencia de la Junta de Andalucía, el Programa Séneca- enfocados hacia un seguimiento estrecho, un marcaje férreo y atosigante de todos y cada uno de los movimientos producidos por cualquier miembro de la comunidad educativa, lo cual está reduciendo, de modo ostensible, la maniobrabilidad de un profesorado cada vez menos identificado con una labor que ya no reconoce como propia.

De la Escuela del Espectáculo a la Escuela Transcultural A pesar de todos los condicionamientos antes analizados, creo que es posible imaginar - con Bradbury - una alternativa de resistencia a la arrogante $y$ 
narcisista acomunicabilidad del "ciberhéroe". Pensemos en esos hombreslibro, en esos hombres-fragmento, hechos de trazos de discursos que siempre remiten a otros discursos, que representan, así, el horizonte alentador de una liberación indefinidamente aplazada, retrotraída a un nuevo empezar contrario a cualquier pretensión de totalidad terminal:

... lo más importante que debíamos meternos en la cabeza contesta uno de ellos al protagonista disidente Montag - es que no somos importantes, que no debemos sentirnos superiores a nadie en el mundo. Sólo somos sobrecubiertas para libros, sin valor intrínseco. (Bradbury, 2005, p.163)

Qué maravillosa metáfora para nosotros. La práctica de un pensamiento disidente, es decir, transgresor, nómada, que se cuestiona y se interroga en todo momento -como el que he tratado de testimoniar en mi exposición- no se marca otros objetivos que la búsqueda incesante de las vías de amplificación de esas desviaciones y contradicciones constitutivas (y constituyentes) del Sistema. Pienso que - sin menospreciar el papel intercesor que puede desempeñar un uso bien temperado de las TIC - es posible otra Escuela, al menos como proyecto siempre abierto que se sobrepasa a sí mismo en su indefinida incompletud. Opuesta a los binarismos epistemológicos, ético-políticos y culturales occidentales desde los que se pronuncia el Capital segmentando y jerarquizando, esta otra Escuela sería fruto de la dinamización positiva y transformadora de la dimensión potencial y posibilitante de esa "brecha discursiva" que Bernstein localiza en la tensión entre lo pensable y lo impensable, entre lo posibleexistente y lo (im)posible-futuro; hecho que, a mi entender, sólo es posible sustituyendo el frenesí alienador de la mera transmisión obsesiva por la intensidad productiva y constituyente del (con)tacto cara a cara.

Si de lo que se trata es de luchar contra la gran Predicción Tecnocrática, admitamos que «las prácticas escolares deben concebirse como contrahegemónicas respecto a los usos culturales de las tecnologías» [San Martín, 1998: 109]. Esto, siempre que seamos capaces de proyectarnos en las líneas múltiples y heterogéneas de una ipseidad compleja y compuesta, en construcción permanente en y a través de esa alteridad, que nos conforma desplazándonos y desemplazándonos para volvernos a emplazar, que nos configura enviándonos fuera de nosotros mismos, diseminándonos en el encuentro híbrido y mestizo con el "otro" (Silva y Browne, 2004). Hay que redirigir la atención desde ese pathos tecnológico recursivo, desde ese falso deleite acomunicacional (y antimediador), el cual sólo remite a su propia fantasmagoría simuladora (siempre anticipada), hacia la reinvención de unos medios heterogéneos, verdaderamente transitivos y mediadores, como los no-lugares la tensión permanente entre unas experiencias nunca consumadas como saberes concluyentes, sino recreadas, de forma constante, en esa apertura a la experiencia incitada por la experiencia misma (Gadamer, 1988).

Ello significaría una radical sustitución del virtualismo como simulacro por la virtualidad como el espacio dinámico de una posibilidad futurizada 
en el plano coral de una temporalidad micronarrativamente multidireccional. Esta virtualidad, asentada en el intercambio comunicativo (y la fertilidad cruzada) con la "otredad", nos recobraría de nuestro espectacular aislamiento iconocrático al suponer una continua redescripción del mundo a partir de la apertura a la novedad de lo diferente. La transculturalidad, tal y como la he definido en otro lugar (Vidal, 2005), sería, en definitiva la base de la destrivialización de una Escuela imprevisible, realizada en el ENTRE, o, si queremos, en el TRANS de una reapropiación recíproca y dialógica de un ser-posibilidad apto para pensar lo impensable, para decir lo indecible, para realizar lo irrealizable, más allá del espacio normalizado y desfuturizador de los Medios Soberanos. Señalo, en suma, hacia una Escuela Transcultural donde la escucha del "otro" y de lo "otro", donde la recepción efectual de la palabra y el gesto representen la elocuencia de un silencio por fin roto.

\section{Referencias}

APPLE, M. W. Educación y poder. Barcelona: Paidós, 1997.

BAUDRILLARD, C. Crítica de la economía política del signo. Madrid: Siglo XXI, 1989.

BAUDRILLARD, C. El crimen perfecto. Barcelona: Anagrama, 1996.

BERNSTEIN, B. Poder, educación y conciencia: sociología de la transmisión cultural. Barcelona: EI Roure, 1990.

BRADBURY, R. Fahrenheit 451. Barcelona: Debolsillo, 2005.

COLINA, C. E. McLuhan y las tecnologías de la comunicación. HUMÁNITAS. Portal temático en Humanidades. 2005. Disponível em:<http://www.revele.com.ve/pdf/anuario_ininco/vol1-n5/ pag173.pdf>. Acesso em: 03 mai. 2005.

DEBORD, G. La sociedad del espectáculo. Valencia: Pre-Textos, 2002.

FOUCAULT, M. Saber y verdad. Madrid: Las Ediciones de La Piqueta, 1991.

FOUCAULT, M. Historia de la sexualidad. 1. La voluntad de saber. Madrid: Siglo XXI, 1992.

GADAMER, H. G. Verdad y método: fundamentos de una hermenéutica filosófica. Salamanca: Sígueme, 1988.

GUERREAU, A. El Feudalismo: un horizonte teórico. Barcelona: Crítica, 1984.

HARDT, M.; NEGRI, A. Imperio. Traducción: Eduardo Sadier de la edición de Harvard University Press, Cambridge, Massachussets, 2000 [en línea] Chile Vive: una página abierta a las utopías... Disponível em: <http://www.chilevive.cl.>. Acesso em: 6 dez. 2003.

LÓPEZ GARCíA, Á. En medio de los medios. Zaragoza: Las Tres Sorores-PRAMES, 1998.

MICHÉA, J. C. La escuela de la ignorancia. Madrid: Acuarela Libros, 2002.

NIETZSCHE, F. Sobre verdad y mentira en sentido extramoral. Madrid: Tecnos, 1994.

OSSET, J. Las nuevas tecnologías en ciencias sociales: un viaje hacia el aula virtual. Íber. Didáctica de las Ciencias Sociales, Geografía e Historia, n.41, p.57-67, 2004.

SAN MARTíN, Á. El compromiso moral y político de la educación en la sociedad de la información. In: PONS, J. P.; SEGURA, J. J. (Coord.). Nuevas tecnologías: comunicación audiovisual y educación. Barcelona: Cedecs, 1998. p.103-21. 
SILVA, V.; BROWNE, R. Escrituras híbridas y rizomáticas: paisajes intersticiales, pensamiento del entre, cultura y comunicación. Sevilla: Arcibel, 2004.

VIDAL, R. Identidad, poder y conocimiento en la sociedad de la información: introducción al estudio de la temporalidad como eje del análisis hermenéutico. 2003. Alicante: Biblioteca Virtual Miguel de Cervantes [en línea]. Disponível em: <http://www.cervantesvirtual.com/FichaObra.html?Ref=11545> Acesso em: 1 mai. 2004.

VIDAL, R. El "otro" como enemigo. Identidad y reacción en la nueva "cultura global del miedo",

Nómadas. Rev. crít. Cienc. Sociales y Jurídicas [en línea], n.9, enero-junio de 2004. Disponível em: <http://www.ucm.es/info/eurotheo/nomadas/>. Acesso em: 12 out. 2004.

VIDAL, R. Hermenéutica y Transculturalidad. Propuesta conceptual para una desconstrucción del "multiculturalismo" como ideología. Redes. Com. Rev. Estud. Desarrollo Soc. Comunic. Instit. Eur. Comunicac. Desarrollo, n.2, p.49-68, 2005.

VIRILIO, P. La máquina de visión. Madrid: Cátedra, 1989.

VIRILIO, P. El cibermundo, o la política de lo peor. Madrid: Cátedra, 1997.

VON FOERSTER, H. Las semillas de la cibernética: obras escogidas. Barcelona: Gedisa, 1996.

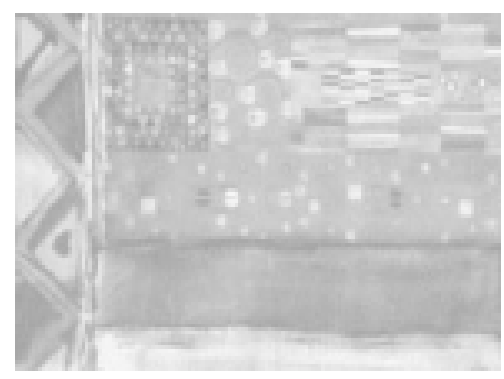

JIMÉNEZ, R. V. Educação, poder e mercado: desconstrução crítica dos efeitos disciplinantes das TIC na nova Escola do Espetáculo. Interface - Comunic., Saúde, Educ., v.9, n.18, p.475-88, set/dez 2005.

Sem negar as inquestionáveis contribuições metodológicas que podem representar as TIC - no contexto de adaptação do ensino às mudanças históricas que estão tendo lugar, esta comunicação pretende ser um convite à reflexão auto-crítica das possíveis conseqüências sócio-cognitivas de determinado uso “abusivo" das mesmas. Basicamente, trata-se de analisar o modo pelo qual isso poderia derivar no triunfo de uma nova cultura afásica: uma cultura sem palavras, em que o "hiperrealismo" enganoso da Imagem onipresente implica uma destruição (iconocrática e iconofágica) da linguagem verbal como mediador não só do processo de ensino-aprendizagem, mas também das relações do sujeito-aluno consigo mesmo e com os demais. A partir do enfrentamento responsável da situação pósmoderna, o que aqui se questiona, definitivamente, é a implantação crescente de uma espécie de ensino-espetáculo, de um ensino reduzido ao simples Simulacro (consumista) de si, na perda da Palavra como veículo de um pensamento capaz de marcar distâncias com respeito aos diagramas normalizadores do novo Capitalismo (Disciplinar) de Redes $e$ a Cultura (Global) do Medo.

PALABRAS-CHAVE: tecnologias de informação e comunicação (TIC). ensino-aprendizagem. cultura afásica. linguagem verbal. 